\title{
Modeling Charge Redistribution at Magnetite Interfaces in Empirical Force Fields
}

\author{
Mine Konuk, Kai Sellschopp, Gregor B. Vonbun-Feldbauer, and Robert H. Meißner*
}

Cite This: J. Phys. Chem. C 2021, 125, 4794-4805

Read Online

ABSTRACT: Magnetite shows enormous potential from biocompatible hybrid materials to heterogeneous catalysis. However, a detailed atomistic understanding of magnetite in complex nanostructures and at interfaces is required to unfold these potentials. Methods capable of treating (several) thousands of atoms and achieving an optimal balance between accuracy and efficiency are therefore in great demand. Here, a new empirical force field for the (001) and (111) magnetite surfaces is developed using partial point charges derived from $a b$ initio Bader charge analyses. An accurate description of electrostatic interactions enables the modeling of magnetite-organic and magnetite-water interfaces. Consequently, surface charge redistribution is proposed as the most relevant mechanism for the surface reconstruction of magnetite and the bidentate binding of ligands. The produced force

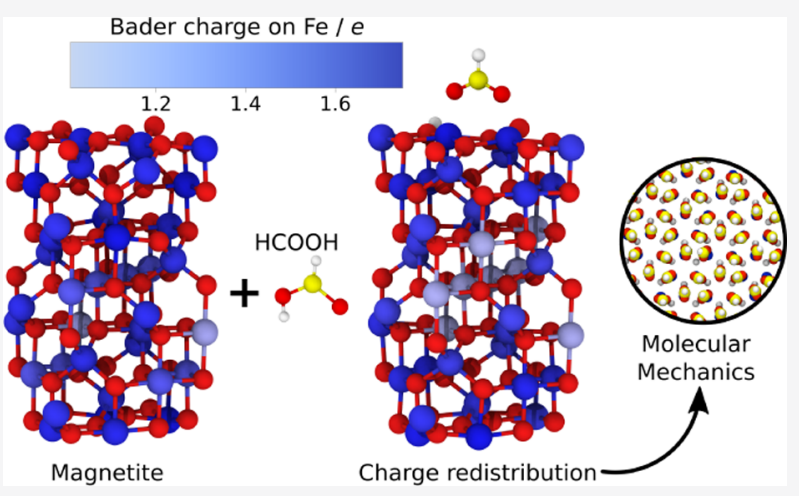
field results are in excellent agreement with the latest findings on magnetite. The approach can be further applied to magnetite nanoparticles and easily extended to oxide and other ionic crystal surfaces.

\section{INTRODUCTION}

Naturally occurring composite materials such as bone, teeth, and nacre consist of unique hierarchical combinations of hard and soft phases of inorganic and organic materials, respectively, in length scales ranging from nano- to micrometres. ${ }^{1}$ Although a complete understanding of these complex systems in nature is not yet acquired, bioinspired materials have exhibited advancements to reach the desired hierarchical structuring. ${ }^{2,3}$ In a recent attempt to provide a new class of bioinspired ceramic materials, self-organization of magnetite $\left(\mathrm{Fe}_{3} \mathrm{O}_{4}\right)$ nanoparticles functionalized with oleic acid molecules leads to a hierarchically arranged well-ordered superstructure with exceptional mechanical properties. $^{4-6}$ Furthermore, organically functionalized magnetite nanoparticles are relevant for the delivery of pharmaceuticals or for theranostatic applications ${ }^{7}$ and the removal of contaminants in water. ${ }^{8}$ The understanding of surface properties of magnetite such as interactions between adsorbates and magnetite surfaces (e.g., adsorbate-induced surface reconstruction), ${ }^{9-11}$ the type of adsorption (molecular or dissociative), ${ }^{12-15}$ and the effects of surface functional groups on the formation of magnetite nanoparticles ${ }^{16}$ provided a route to control the growth and stability of hierarchical magnetite nanocomposites. The structure and electronic properties of recently experimentally observed magnetite surfaces have a major influence on the growth and stability of such nanocomposites, and the observed surface structures typically differ significantly from surfaces of a (theoretical) straightforward cleaving of $\mathrm{Fe}_{3} \mathrm{O}_{4}$.

Many experimental and theoretical studies on the reconstruction and stabilization of magnetite surfaces have been performed to get an insight into surface structures and reconstructions associated with the local electronic structure. ${ }^{17}$ Among them, $\{001\}$ magnetite surfaces terminated by octahedral coordinated iron $\left(\mathrm{Fe}_{\text {oct }}\right)$ and oxygen have been one of the most commonly studied surface terminations of $\mathrm{Fe}_{3} \mathrm{O}_{4}$. The clean $(001)$ surface shows a $(\sqrt{2} \times \sqrt{2}) R 45^{\circ}$ reconstruction in experiments, which was first explained by a distorted bulk truncation (DBT) model based on density functional theory (DFT) calculations. ${ }^{18}$ However, later, this model was replaced by the subsurface cation vacancy (SCV) structure, where two vacancies are formed at octahedral iron positions in the subsurface and an additional interstitial iron appears in the surface $\mathrm{Fe}_{\text {tet }}$ layer. ${ }^{9,19}$ Both structures are shown in Figure 1 along with a typical $\mathrm{Fe}_{\text {tet1 }}$-terminated magnetite (111) surface.

For a magnetite (111) surface, six different polar bulk terminations are possible according to Tasker's rules. However, $\mathrm{Li}$ and Paier ${ }^{20}$ showed that only two of them, the $\mathrm{Fe}_{\text {tet1 }}$ and $\mathrm{Fe}_{\text {oct2 }}$ terminations, are stable in a wide range of oxygen chemical

Received: November 17, 2020

Revised: January 29, 2021

Published: February 17, 2021

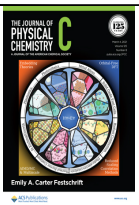




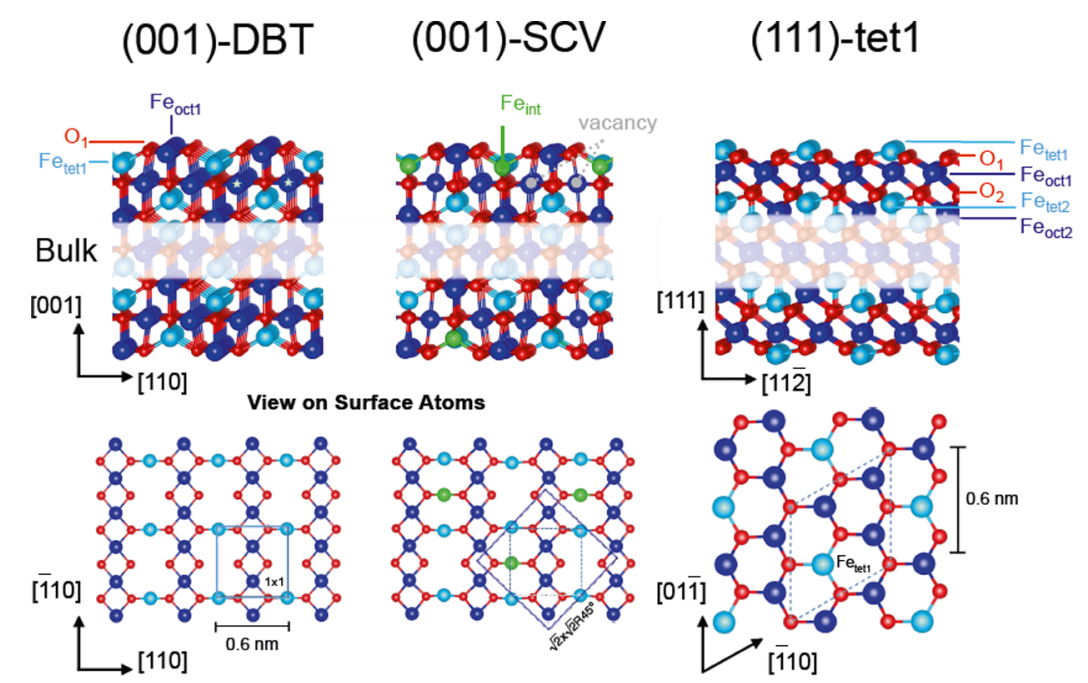

Figure 1. Magnetite surface structures. On (001) and on (111) surfaces, a set of three atomic layers of $\mathrm{Fe}_{\text {oct }} \mathrm{O}$, and $\mathrm{Fe}_{\text {tet }}$ are referred to as a surface, subsurface, sub-subsurface or bulk layer, respectively. The bulk region of the (111) surface is, however, defined by a set of five atomic layers (i.e., $\mathrm{Fe}_{\text {tet } 1}-$ $\left.\mathrm{O}_{1}-\mathrm{Fe}_{\text {oct1 }}-\mathrm{O}_{2}-\mathrm{Fe}_{\text {tet2 }}-\mathrm{Fe}_{\text {oct2 }}\right)$. We use the following color scheme: light blue for $\mathrm{Fe}_{\text {tet }}$, dark blue for $\mathrm{Fe}_{\text {oct }}$, red for oxygen, yellow for carbon, and white for hydrogen. The interstitial iron $\mathrm{Fe}_{\text {int }}$ and subsurface octahedral iron vacancies appearing upon the formation of the SCV reconstruction are marked with green and gray spheres, respectively. Throughout this work, the same color code is used.

potentials. DFT calculations and experimental studies furthermore agree that an $\mathrm{Fe}_{\text {tet } 1}$-terminated magnetite (111) surface is present in typical ultrahigh vacuum and also more oxidizing conditions for both single crystal surfaces and thin films (cf. Figure 1). ${ }^{21-25}$ Beyond that, both $\mathrm{Fe}_{\text {tet } 1}$ and $\mathrm{Fe}_{\text {oct } 2}$ terminations of the $\mathrm{Fe}_{3} \mathrm{O}_{4}(111)$ surface are oxygen-rich and provide multiple sites for the adsorption and dissociation of small molecules such as $\mathrm{H}_{2}$ or $\mathrm{O}_{2},{ }^{26}$ water, ${ }^{20,27}$ methanol, ${ }^{28,29} \mathrm{CO}_{2},{ }^{30,31}$ or $\mathrm{CO}{ }^{32}$ The adsorption of hydrogen ${ }^{33}$ and formic acid ${ }^{10,34}$ was investigated in detail both experimentally and computationally for the magnetite (001) surface as well. Particularly interesting is that the SCV reconstruction on magnetite is lifted by the dissociative formic acid adsorption. ${ }^{10,34}$

Water adsorption on the $\mathrm{Fe}_{3} \mathrm{O}_{4}(001)$ surface, on the other hand, remains still debated. There is some ambiguity about the surface reconstruction of $\mathrm{Fe}_{3} \mathrm{O}_{4}(001)$ upon water adsorption, and whether the reconstruction is favorable or not depends eventually on the water coverage. ${ }^{13,35,36}$ Systematic experimental and theoretical studies on water adsorption on both SCV and DBT magnetite surface structures have shown that at low water pressure, a reconstructed SCV surface is the most stable termination, ${ }^{13}$ while others, however, suggested the formation of a self-limited surface oxyhydroxide phase ${ }^{35}$ or surface roughening. ${ }^{36}$ At a high water coverage, a hydrated DBT magnetite surface structure is energetically the most favorable. ${ }^{14}$

As demonstrated by these examples, understanding the chemical reactivity of $\mathrm{Fe}_{3} \mathrm{O}_{4}$ is challenging due to the complex interfacial chemical behavior of magnetite. One of the ultimate goals for simulating magnetite nanoparticles is, however, to determine the atomistic stability of magnetite surfaces functionalized with biomolecules. Thus, realistic representations of different surface structures of magnetite must be carefully modeled on the basis of biocompatible force-field potentials if they are to be modeled holistically using an empirical force field. However, it is still challenging to model magnetite-organic nanoparticles with empirical force fields using fixed partial point charges due to the lack of accurate approximations of the electronic structure and the complex oxidation state of magnetite. Especially, in a situation where the surface is not completely covered with ligands and/or the mechanical properties of a magnetite nanocomposite are of interest, the use of bulk charges for surface atoms leads to unphysical surface structures (see the first supplementary video in the Supporting Information), which is presumably the reason for a fixation of magnetite atoms in other studies, ${ }^{7,8,37}$ limiting their predictability. Hence, an accurate approximation of the charge distribution is necessary to obtain realistic surface structures and to model accurately the mechanical behavior of magnetitebased nanocomposites. Thanks to advances in computational algorithms and the accuracy of empirical force fields, simulations on atomistic scales have already provided a deeper insight into many other iron oxide-based materials that are not magnetitebased. ${ }^{38-40}$ To our knowledge, only one approach has been followed so far to simulate magnetite interfaces with water and formic acid using formal charges for magnetite. ${ }^{41}$ Formal charges, however, prohibit the use of established biomolecular force fields and thus require an extensive parameterization for each new ligand. In contrast to fixed partial charge approaches, charge equilibriation $\left(Q_{e q}\right)$ approaches applied to magnetite are typically incompatible with biomolecular force fields ${ }^{42}$ and behave poorly when reproducing the required standard charges $(\mathrm{RESP})^{43}$ used in biomolecular force fields for magnetite. Hence, in this work, atomic scale simulations based on DFT calculations and empirical force fields were used to study the structural and electronic properties of the interface between small biomolecules and magnetite surfaces. The results of the simulations are presented and analyzed, and a brief outlook on possible applications of a newly developed empirical magnetite force field is presented.

We divided our approach to parameterize an empirical force field for magnetite into three parts. In the first part, a Bader charge analysis ${ }^{44,45}$ for each of the two DBT and SCV structures on the (001) and $\mathrm{Fe}_{\text {tet1 }}$-terminated (111) magnetite surface is carried out to investigate structural properties originating from charge redistributions on atoms in surface layers. In the second part, the dissociation of formic acid on magnetite (001) and (111) is discussed and an improved empirical force field parameterization for magnetite surfaces is developed utilizing 
point charges that are determined from atomic Bader charges for different bare surface structures and with adsorbed organic molecules. By adapting relative charge differences calculated from DFT in our empirical force field, stable SCV-, DBT-, and $\mathrm{Fe}_{\text {tet1 } 1}$-terminated (111) surfaces at $300 \mathrm{~K}$ are obtained in $\mathrm{MD}$ simulations. As a validation and benchmark of our parameterization for interfaces with organic molecules, we discuss energetic and structural differences of formic acid-covered surfaces using the new force field and compare those with the corresponding calculations from DFT. In the third part, we examine the adsorption behavior of water on $\mathrm{Fe}_{3} \mathrm{O}_{4}(001)$ surfaces. Different morphologies of water molecules at different coverages on magnetite surfaces are analyzed and brought into relation with previous experimental results and theoretical calculations from the literature. Additionally, the heats of immersion of DBT and SCV surface structures are presented using the new empirical force field and compared to experimentally determined values.

\section{METHODS}

Electronic Structure Calculation. All DFT calculations presented here are performed using the Vienna $\mathrm{Ab}$ initio Simulation Package (VASP, version 5.4.4). ${ }^{46,47}$ The plane-wave code utilizes periodic boundary conditions and projector augmented-wave pseudopotentials ${ }^{48}$ which were chosen to model the interactions between the ionic cores and the valence electrons. To ensure accurate results, energy convergence with respect to the energy cutoff for the plane-wave basis, the thickness of vacuum region decoupling surface slabs, and the $k$ point sampling were carefully tested to be within $1 \mathrm{meV}$. The following values are chosen in order: $520 \mathrm{eV}$ energy cutoff, $16 \AA$ of vacuum, and $5 \times 5 \times 1$ and $7 \times 7 \times 1$ Monkhorst-Pack grids for the (001) and (111) surfaces, respectively. The number of atomic layers in the $\mathrm{Fe}_{3} \mathrm{O}_{4}$ surface slabs was set to be 13 and 17 for the (001) and (111) surfaces, respectively. However, further convergence tests were also carried out on thicker slabs. The PBE exchange-correlation functional was used for all calculations. ${ }^{49}$ To correct the significant correlation effects of the Fe d-electrons, an on-site Hubbard parameter $U_{\text {eff }}$ of $4 \mathrm{eV}$ was used by employing the method of Dudarev et al., ${ }^{50}$ which is known to accurately reproduce the structure, magnetic moments, and band gap of magnetite. ${ }^{51,52}$ More details on the computational setup and the model system can be found in previous publications. ${ }^{34,53}$ To calculate energy-displacement curves for organic molecules and $\mathrm{Fe}_{3} \mathrm{O}_{4}$ surfaces, energetically favorable configurations from previous studies were used ${ }^{34,53}$ as initial structures. The distance between the adsorbates and the surfaces was varied, and a static calculation was performed for every resulting configuration. Partial charges on atoms can be determined with a Bader charge analysis: ${ }^{44}$ From the calculated electronic charge densities, a minimum in the charge density between the atoms is typically found, which allows the partitioning of the electronic charge density and assigning it to specific atoms. Although partial atomic charges obtained from approaches to partition the electron density are not observable and their interpretation in terms of oxidation states is intrinsically ambiguous, ${ }^{54}$ they do provide a measure to keep track of relative charge transfer. ${ }^{5,56}$

The Bader analysis is using the corresponding code (version 1.00) from the Henkelman group ${ }^{45}$ and adapts their specific hints for VASP users.

A two-step restrained electrostatic potential fit charge model $^{43}$ (RESP) was used to obtain partial point charges for formic acid and formate which reproduce the electrostatic potential in QM calculations using the B3LYP functional method, a polarizable water model (c-PCM), and a cc-pV( $\mathrm{T}$ $+d) Z$ basis set. To obtain partial point charges practically, the Small-molecule Topology GEnerator $(\mathrm{STaGE})^{57}$ was used, which has a built-in RESP implementation and calculates the desired charges from simple SMILES representation of formate (i.e., $\mathrm{C}(=\mathrm{O})[\mathrm{O}-])$ and formic acid (i.e., $\mathrm{C}(=\mathrm{O}) \mathrm{O})$.

Molecular Dynamics Simulations. All MD simulations have been performed using LAMMPS. ${ }^{58}$ A Nosé-Hoover thermostat and barostat are utilized to retain systems in equilibrium at distinct temperatures and pressures. Equations of motion are integrated with steps of $0.5 \mathrm{fs}$ using the velocityVerlet algorithm. The total energy of the system is composed by two types of interactions: intramolecular and intermolecular. The former determines the energy for holding atoms together within a molecule, while the latter defines interactions that occur between molecules. In an ionic solid, such as magnetite, the intermolecular interactions influence mainly the system's behavior where intramolecular interactions are typically small. The interaction energy between two atoms of magnetite is thus well described by a short-ranged repulsive potential and electrostatic Coulomb interactions between each pair of atoms $i$ and $j$ with atomic charges $q_{i}$ and $q_{j}$ separated by a distance $r_{i j}$

$$
E_{\text {non-bonded }}=\sum_{i j} 4 \epsilon_{i j}\left[\left(\frac{\sigma_{i j}}{r_{i j}}\right)^{12}-\left(\frac{\sigma_{i j}}{r_{i j}}\right)^{6}\right]+\sum_{i j}\left(\frac{q_{i j} q_{i j}}{r_{i j}}\right)
$$

the parameters $\epsilon_{i j}$ and $\sigma_{i j}$ for each pair ij of interacting atoms is calculated using standard Lorentz-Berthelot combination rules, $\epsilon_{i j}=\left(\epsilon_{i} \epsilon_{j}\right)^{1 / 2}$ and $\sigma_{i j}=\left(\sigma_{i}+\sigma_{j}\right) / 2$. Lorentz-Berthelot combination rules have been successfully used for decades in standard biomolecular force fields such as $\mathrm{AMBER}^{59}$ or $\mathrm{CHARMM}^{60}$ and have proven to accurately predict the interfaces of minerals, metals, oxides, and (bio)polymers. ${ }^{61-65}$ Initially, in order to simulate bulk magnetite, parameters for the short-ranged repulsive part are adapted from ref 66 which presents interatomic potentials for hematite-water interfaces using a modified CLAYFF ${ }^{63}$ parameter set. CLAYFF ${ }^{63}$ is a common and reliable parameterization for many minerals and provides a reasonable description for metal-oxygen interactions of iron, oxygen, and hydrogen atoms associated with bulk (hydrated) phases. Van der Waals potential parameters for bulk magnetite and its interfaces used in this work are summarized in Table 1.

By simply ensuring a charge neutral bulk crystal of magnetite, the charge for the octahedral iron in magnetite was estimated to be $1.313 \mathrm{e}$. For more complex systems, for example, magnetite surfaces, the redistribution of charges on the surface was taken into account by employing results from a Bader charge analysis calculated in DFT into an empirical force field. Partial point charges of magnetite surface atoms used in the empirical force field are rescaled with

$$
q_{\text {ion,layer }}^{\mathrm{FF}}=q_{\text {ion, bulk }}^{\mathrm{FF}} \cdot \frac{\left\langle q_{\text {ion }}\right\rangle_{\text {layer }}}{\left\langle q_{\text {ion }}\right\rangle_{\text {bulk }}}
$$

where the ion is $\mathrm{Fe}_{\text {oct }}, \mathrm{Fe}_{\text {tet }}$, or $\mathrm{O}$, "layer" indicates the respective atomic layer number, and expressions in brackets $\langle\ldots\rangle_{\text {bulk }}$ and $\langle\ldots\rangle_{\text {layer }}$ indicate either an average over all Bader charges for each ion type of a bulk magnetite crystal or an average of Bader charges over each atomic layer of the magnetite surface slab, respectively. $q_{\text {ion,layer }}^{\mathrm{FF}}$ represents the corresponding partial point 
Table 1. Parameters for the Lennard-Jones Potential and Bonded Interactions to Describe Magnetite in an Empirical Force Field

\begin{tabular}{|c|c|c|}
\hline nonbonding & $\epsilon_{i j} / \mathrm{kcal} \mathrm{mol}^{-1}$ & $\sigma_{i j} / \AA$ \\
\hline iron in the octahedral site $\left(\mathrm{Fe}_{\mathrm{oct}}\right)$ & $9.0298 \times 10^{-6}$ & 4.0722 \\
\hline iron in the tetrahedral site $\left(\mathrm{Fe}_{\text {tet }}\right)^{a}$ & $9.0298 \times 10^{-6}$ & 4.0722 \\
\hline oxygen $(\mathrm{O})^{a}$ & 0.1554 & 3.1655 \\
\hline hydroxyl oxygen $\left(\mathrm{O}_{\mathrm{H}}\right)^{a}$ & 0.1554 & 3.1655 \\
\hline hydroxyl hydrogen $\left(\mathrm{H}_{\mathrm{O}}\right)^{a}$ & 0.0000 & 0.0000 \\
\hline water oxygen $\left(\mathrm{O}_{\mathrm{W}}\right)^{b}$ & 0.1020 & 3.1880 \\
\hline water hydrogen $\left(\mathrm{H}_{\mathrm{W}}\right)^{b}$ & 0.0000 & 0.0000 \\
\hline bond stretch & $k_{\text {bond }} / \mathrm{kcal} \mathrm{mol}^{-1} \AA^{-2}$ & $r_{\text {eq }} / \AA$ \\
\hline $\mathrm{O}_{\mathrm{H}}-\mathrm{H}_{\mathrm{O}}^{c}$ & 554.1349 & 1.0000 \\
\hline $\mathrm{O}_{\mathrm{W}}-\mathrm{H}_{\mathrm{W}}^{b}$ & 450.0000 & 0.9572 \\
\hline angle bend & $k_{\theta} / \mathrm{kcal} \mathrm{mol}^{-1} \mathrm{rad}^{-2}$ & $\theta_{\text {eq }} / \operatorname{deg}$ \\
\hline $\mathrm{Fe}_{\mathrm{oct}}-\mathrm{O}_{\mathrm{H}}-\mathrm{H}_{\mathrm{O}}^{c}$ & 30.0000 & 109.4700 \\
\hline $\mathrm{H}_{\mathrm{W}}-\mathrm{O}_{\mathrm{W}}-\mathrm{H}_{\mathrm{W}}^{b}$ & 55.0000 & 104.5200 \\
\hline $\begin{array}{l}a_{\text {Taken from }} \text { Kerisit. }^{66} b_{\text {Take }} \\
\text { CLAYFF. }\end{array}$ & ${ }^{b_{\text {Taken }}}$ from TIP3P. ${ }^{67}$ & ${ }^{{ }^{c}}$ Taken from \\
\hline
\end{tabular}

charge in the empirical force field. $q_{\text {ion,bulk }}^{\mathrm{FF}}$ represents the bulk crystal partial charges of magnetite. Within this formalism, partial point charges are adjusted under the constraint of a total charge neutral system. The remaining residual charges due to nonstoichiometry of resulting surface slabs in the order of approximately $\pm 1-2 e$ per surface unit cell are thus distributed over all atoms in the surface and subsurface layers of the slab.

An empirical force field compatible with well-established biomolecular force fields, such as the General AMBER force field $\left(\mathrm{GAFF}^{68}\right)$, was achieved by rescaling partial point charges for magnetite-organic interfaces as follows: (i) bare magnetite surface partial point charges are the starting point for all atoms in the slab. (ii) Surface atoms that are in the vicinity of the formic acid $(\mathrm{HCOOH})$ dissociation reaction into formate $\left(\mathrm{HCOO}^{-}\right.$ and $\mathrm{H}^{+}$) are rescaled with their corresponding Bader charges and using eq 2 (see highlighted atoms in Figure 3). According to the Bader charge analysis, relevant affected magnetite atoms are surface hydroxyl group oxygens, $\mathrm{O}_{\mathrm{H}}$, octahedral iron atoms bound to a surface hydroxyl group oxygen, $\mathrm{Fe}_{\mathrm{oct} 2}\left(-\mathrm{O}_{\mathrm{H}}\right)$, and iron atoms bound to a carboxylate anion oxygen, $\mathrm{Fe}_{\text {oct1 }}\left(-\mathrm{O}_{\mathrm{A}}\right)$ or $\mathrm{Fe}_{\text {tet } 1}\left(-\mathrm{O}_{\mathrm{A}}\right)$. In the case of a single water molecule dissociating into two surface hydroxyl groups (cf. Figure S2d), a partial charge of $-0.950 e$ for $\mathrm{O}_{\mathrm{H}}$ and $+0.425 e$ for $\mathrm{H}_{\mathrm{O}}$ on both surface hydroxyl groups are used. Upon dissociation of water, small residual charges - around $0.055 e$ for (001)-SCV and $0.049 e$ for (001)-DBT per surface unit cell-are equally distributed to iron atoms connected to the resulting hydroxyl oxygen. All partial point charges used in the empirical force field for the different surfaces and interfaces are summarized in Tables S3 and S4.

The energy between covalently bonded atoms in the organic molecules formed by bonds, angles, and dihedrals is given by the standard functional form of bonded interactions in many organic force fields

$$
\begin{aligned}
E_{\text {bonded }}= & \sum_{\text {bond }} k_{\text {bond }}\left(r-r_{\text {eq }}\right)^{2}+\sum_{\text {angle }} k_{\theta}\left(\theta-\theta_{\text {eq }}\right)^{2} \\
& +\sum_{\text {dihedral }} k_{\phi}[1+\cos (n \phi-\delta)]
\end{aligned}
$$

The first term represents a harmonic bond where $k_{\text {bond }}$ is a force constant and $r_{\text {eq }}$ is the equilibrium bond length. Angles are described by simple harmonic relationship as well, where $k_{\theta}$ is a force constant and $\theta_{\mathrm{eq}}$ is the equilibrium bond angle. Dihedrals in a bonded four-atom chain are described by a periodic cosine term with parameters $k_{\phi}, n, \phi$, and $\delta$. It should be noted that the hydroxyl groups on the surface are modeled as well using a harmonic bond and angle, as given in eq 3, according to the CLAYFF formalism.

A standard Ewald summation with a precision of $10^{-6}$ and a cutoff of $12 \AA$ is used to solve the long-range electrostatic interactions. To generate a sufficient vacuum separation in our calculations, the slab keyword in LAMMPS is used to tackle problems emerging from periodic interactions and dipole moments in an infinite array of periodic images. In contrast to the suggested value of 3 for the slab command in LAMMPS, a large vacuum of $10^{7}$ times the length of the unit cell in the nonperiodic dimension was required to converge to the DFT results for slab simulations in vacuum.

Water is modeled by employing the optimized TIP3P parameter $^{67}$ developed for use with a long-range solver, for example, an Ewald summation, for electrostatic interactions. For all simulations including water, water molecules are added to the SCV and DBT surface models with a dimension of $50.6 \times 50.6 \AA^{2}$ in $x$ and $y$ directions and a slab thickness of $23.2 \AA$ along the $z$ direction. In order to test the empirical force field against the available experimental values, the heat of immersion of the magnetite surface was computed. The heat of immersion, $E_{\text {him }}$, is defined as the energy obtained by wetting the dry surface with liquid water. ${ }^{55,69,70}$ For these calculations, the system containing two hydroxylated magnetite surfaces separated by a distance of $118 \AA$ was filled with 10,384 liquid water molecules (see Figure S3 in the Supporting Information). The entire system was simulated for $1 \mathrm{~ns}$ in an $\mathrm{NpT}$ ensemble at $1 \mathrm{~atm}$ in which a barostat was only applied to the direction normal to the surface slab. The resulting system was equilibrated for additional $1 \mathrm{~ns}$ in an NVT ensemble at $300 \mathrm{~K}$. The water density in a central region far away from the surfaces automatically adjusts itself during the $N p T$ run to $0.997 \mathrm{~g} /$ $\mathrm{cm}^{3}$, which is the bulk equilibrium value provided by TIP3P ${ }^{67}$ (Figure S3 in the Supporting Information). Room temperature heats of immersion $\left(E_{\text {him }}\right)$ were calculated from the difference between the total energies of the entire system in contact with water $\left(E_{\text {tot }}\right)$ and total energies of the corresponding hydroxylated but dry surface slab in vacuum $\left(E_{\text {surf }}\right)$ and a bulk water system $\left(E_{\text {wat }}\right)$ containing the same amount of water

$$
E_{\text {him }}=\left(E_{\text {tot }}-\left(E_{\text {wat }}+E_{\text {surf }}\right)\right) /\left(2 A_{\text {surf }}\right)
$$

$A_{\text {surf }}$ is the surface area of one side of the magnetite slab. The corresponding average energies were obtained in separate $\mathrm{MD}$ simulations at $300 \mathrm{~K}$ for $1 \mathrm{~ns}$ where energies were collected every 0.5 ps. Adsorption energies per water molecule are calculated from

$$
E_{\mathrm{ads}}=\left(E_{n\left(\mathrm{H}_{2} \mathrm{O}\right) / \mathrm{Fe}_{3} \mathrm{O}_{4}(001)}-E_{\mathrm{Fe}_{3} \mathrm{O}_{4}(001)}-n E_{\left(\mathrm{H}_{2} \mathrm{O}\right)}\right) / n
$$

and

$$
\begin{aligned}
E_{\mathrm{ads}}^{*}= & \left(E_{n\left(\mathrm{H}_{2} \mathrm{O}\right) /\left(\mathrm{OH} @ \mathrm{Fe}_{3} \mathrm{O}_{4}(001)\right)}-E_{\mathrm{OH} @\left(\mathrm{Fe}_{3} \mathrm{O}_{4}(001)\right)}\right. \\
& \left.-n E_{\left(\mathrm{H}_{2} \mathrm{O}\right)}\right) / n
\end{aligned}
$$

for the bare surfaces and surfaces already covered with $\mathrm{OH}$ (mixed adsorption mode), respectively. 


\section{RESULTS AND DISCUSSION}

Bare $\mathrm{Fe}_{3} \mathrm{O}_{4}(001)$ and (111) Surfaces. Magnetite has an inverse spinel structure, $1 / 3$ of the iron ions $\left(\mathrm{Fe}^{\mathrm{III}}\right)$ are tetrahedrally and $2 / 3$ of the iron ions $\left(\mathrm{Fe}^{\mathrm{II}, \mathrm{III}}\right.$ in the ratio $\left.1: 1\right)$ are octahedrally coordinated by oxygen. Two main ideas are considered here in order to simulate bulk $\mathrm{Fe}_{3} \mathrm{O}_{4}$ using an empirical force field: (i) bulk magnetite is typically above the Verwey temperature in our simulations and each $\mathrm{O}^{2-}$ is thus thought of as being coordinated to three octahedral $\mathrm{Fe}^{2.5+}$ ions and one tetrahedral $\mathrm{Fe}^{3+} \cdot{ }^{71}$ At room temperature, it is likely for the electron to hop between the $\mathrm{Fe}^{\mathrm{II}}$ and $\mathrm{Fe}^{\mathrm{III}}$ atoms on octahedral sites, ${ }^{72}$ resulting effectively in a formal charge of +2.5 on $\mathrm{Fe}_{\text {oct }}$ atoms. (ii) Modified CLAYFF parameters ${ }^{66}$ - originally developed to describe hematite and water-are used as a basis for our new force field. CLAYFF parameters are modified in such a way that partial charges of iron are fixed to +1.574 e for tetrahedral sites and to $+1.313 e$ for octahedral sites, while oxygen still has $-1.050 e$ in bulk magnetite (more details are found in Methods). To avoid confusion, $e$ refers, in the whole manuscript, to the positive elementary charge. Using this charge modification, structural properties of bulk magnetite are in good agreement with previously reported simulations and experiments and ensure, furthermore, charge neutrality of the bulk crystal model. The calculated lattice parameter is $8.41 \AA$ at $300 \mathrm{~K}$ which is in excellent agreement to the lattice constant of $8.39 \AA$ determined experimentally. ${ }^{73,74}$

Cleaving a bulk $\mathrm{Fe}_{3} \mathrm{O}_{4}$ crystal in the models along the (001) plane encloses the resulting stoichiometric slab in two (001) surfaces, one terminated by a layer of $\mathrm{Fe}_{\text {oct }}$ and oxygen and the other terminated by $\mathrm{Fe}_{\mathrm{tet}}$. In simulations employing bulk partial charges, this leads to a diffusion of iron atoms to the surface and a nonphysical and rather unstructured surface termination at room temperature (see the first supplementary video in the Supporting Information). On the other hand, creating nonstoichiometric slabs that resemble the experimentally observed surface structure of magnetite makes the use of bulk partial charges impossible, since the overall system must be charge neutral in order to correctly treat long-range electrostatic interactions with most $k$-space solvers. Furthermore, a redistribution of charges close to the surface is observed both in DFT $+U$ and experiments. ${ }^{19}$ Hence, we decided to use bulk charges only as a starting point and to modify partial point charges of surface atoms based on Bader charges obtained from DFT to improve the force field further and make it applicable to magnetite surfaces. Two prominent (001) surface structures, that is, SCV and DBT, and an $\mathrm{Fe}_{\text {tet }}$-terminated (111) surface are investigated in the following by evaluating their Bader charge redistribution. Figure 2 summarizes the relative Bader charges in the first few layers of symmetric (001) and (111) surfaces, which are up to 17 atomic layers thick in total. Relative changes in Bader (dashed lines) and partial point charges (solid lines) in the respective region (surface, subsurface, etc., indicated by the layer number) are obtained by dividing the average layer charge of the slab with the bulk crystal charge: $\left\langle q_{\text {ion }}\right\rangle_{\text {layer }} /\left\langle q_{\text {ion }}\right\rangle_{\text {bulk }}$."Ion" refers to either $\mathrm{Fe}_{\text {oct }} \mathrm{O}$, or $\mathrm{Fe}_{\text {tet }}$ and $\langle\ldots\rangle_{\text {layer }}$ represents an average over the atomic layer (cf. Figure 1). Hydrogen is placed via a harmonic bond on the stronger binding oxygen atoms (observed, e.g., in Arndt et al. ${ }^{34}$ ), and consequently, the stronger binding to this adsorption site is implicitly included through a localized change in the partial charges around this hydrogen (cf. Tables S3 and S4). "Bulk" refers to an average over all ions of a certain type in the bulk crystal. Bader charges for bulk $\mathrm{Fe}_{\mathrm{oct}}, \mathrm{Fe}_{\mathrm{tet}}$
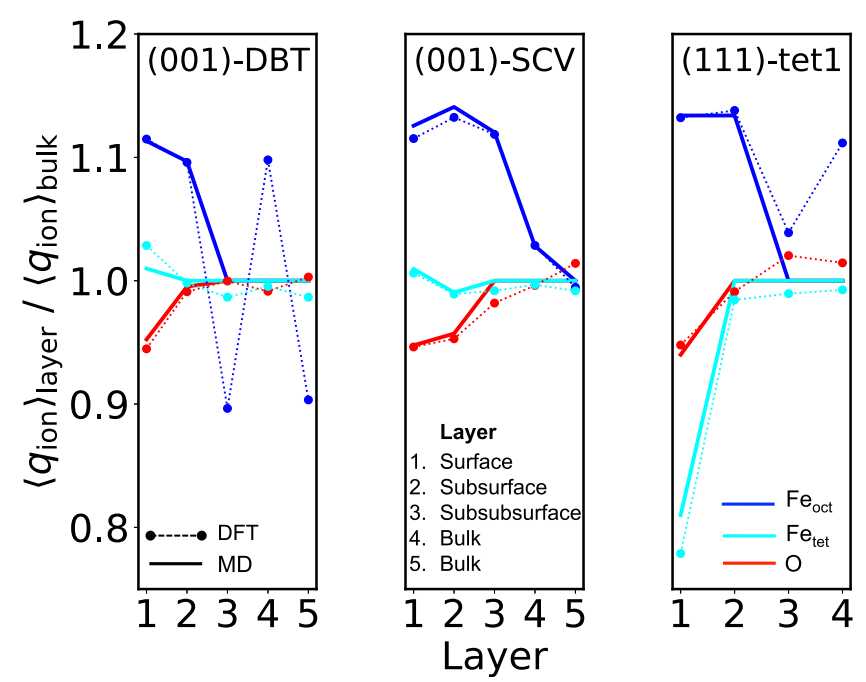

Figure 2. Charge distribution averaged over each atomic species (red$\mathrm{O}$, dark blue $-\mathrm{Fe}_{\text {oct }}$ and light blue- $-\mathrm{Fe}_{\text {tet }}$ ) within surface layers with respect to the atomic layer indices (cf. Figure 1) of DBT (right), SCV (middle), and (111)- $\mathrm{Fe}_{\text {tet1 }}$ (right) surface structures. Partial point charges (solid lines) are derived from Bader charge differences (dashed lines) obtained from DFT calculations. Solid and dashed lines are drawn into the figure only to guide the eye and have no physical meaning.

and oxygen result in $+1.50 e,+1.65 e$, and $-1.17 e$, respectively. It is worth noting that the observed disproportionation between $\mathrm{Fe}^{\mathrm{II}}$ and $\mathrm{Fe}^{\mathrm{III}}$ Bader charges on the octahedral sites is between 0.2 and $0.3 e$ and is in good agreement with results of Liu et al. ${ }^{75}$

According to Figure 2, $\mathrm{Fe}_{\text {tet }}$-Bader charges remain close to their bulk crystal value, with the only exception of the $\mathrm{Fe}_{\text {tet1 }}$ on a (111) surface. $\mathrm{Fe}_{\text {tetl }}$ gains a significant amount of electrons due to the reduced coordination and resembles thus more an $\mathrm{Fe}^{\mathrm{II}}$ rather than an $\mathrm{Fe}^{\mathrm{III}} \cdot{ }^{21}$ In contrast, $\mathrm{Fe}_{\text {oct }}$ in the first few layers loses electrons on all surfaces. Particularly noteworthy is that for an SCV structure, Bader charges of $\mathrm{Fe}_{\text {oct }}$ atoms are still higher in the third layer than those closer to the surface. Apparently, subsurface iron vacancies shift the charge redistribution further in the bulk direction - this confirms that charges on iron(II,III) sites generally have a higher mobility. ${ }^{21}$ As the thickness of the slabs increases, Bader charges between $+1.38 e$ and $+1.65 e$ are observed at octahedral iron positions (cf. Figure S1), which are presumably associated with discrete $\mathrm{Fe}^{\mathrm{II}}$ and $\mathrm{Fe}^{\mathrm{III}}$ ions. ${ }^{56}$ As a result, Bader charges for $\mathrm{Fe}_{\text {oct }}$ layers on (001)-DBT and (111) surfaces alternate from the third layer depending on the slab thickness. In contrast to the (001)-DBT surface, discrete $\mathrm{Fe}^{\mathrm{II}}$ and $\mathrm{Fe}^{\mathrm{III}}$ octahedral iron sites are found in the same atomic layer in the bulk-like region of a (001)-SCV surface. On surfaces, there are less symmetry constraints than in the bulk, which is why the octahedral sites in the bulk-like layers separate into $\mathrm{Fe}^{\mathrm{II}}$ and $\mathrm{Fe}^{\mathrm{III}}$ oxidation states, in agreement with $\mathrm{Liu}$ and $\mathrm{Di}$ Valentin. ${ }^{76}$ However, since stabilities and energies of the magnetite surfaces with our empirical force field are only marginally affected by the exact location of discrete iron(II,III) sites in the bulk region of the slabs, these are effectively modeled by an atomic partial charge which resembles a formal charge of +2.5 . Tables $S 1$ and $S 2$ summarize all Bader charges for different surface terminations.

Typically, it is not possible to use Bader charges directly in empirical force fields, since they are incompatible to partial point charges used in most empirical force fields, for example, $\operatorname{RESP}^{43}$ 

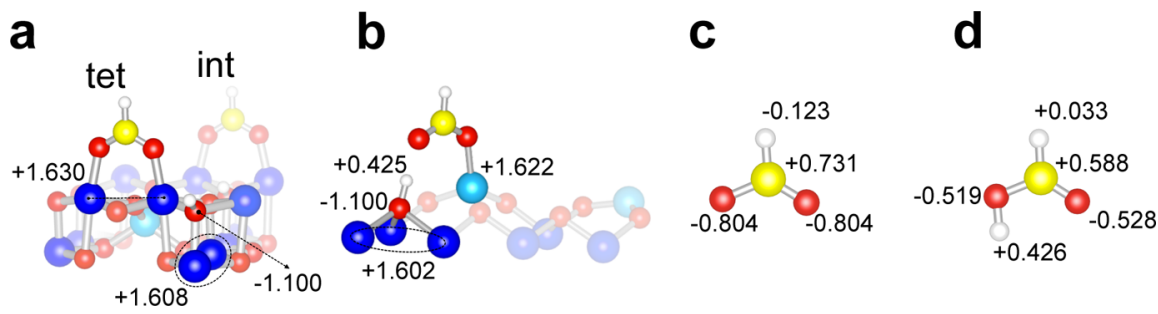

Figure 3. Graphical illustration of the charge model for formic acid adsorption on the magnetite (001) in (a) and the (111) surface in (b). In "tet" and "int" formate adsorption on the unreconstructed (001) magnetite surface, the dissociated hydrogen is attached to an oxygen in an interstitial site. Partial point charges are given in $e$. Partial point charges for a bulk crystal system using our empirical force field have been adapted to $q_{\mathrm{Fe}_{\mathrm{oct}}}=+1.313 e, q_{\mathrm{O}}=-1.050 e$, and $q_{\mathrm{Fe}_{\mathrm{tet}}}=+1.574 e$. For the sake of simplicity, partial charges of the subsurface, sub-subsurface, and bulk-like region have not been shown and can be found in Tables S3 and S4. In (c) and (d), partial point charges from an RESP fit (see Methods) of formate and formic acid molecules are shown. Color code: light blue $-\mathrm{Fe}_{\text {tet }}$ dark blue $-\mathrm{Fe}_{\text {oct }}$ red-oxygen, yellow-carbon, and white-hydrogen.

in GAFF. ${ }^{68}$ To model the charge redistribution between layers in an empirical force field, all surface partial charges have been rescaled based on the relative Bader charge differences and the previously introduced bulk partial charges (see Figure 2 and Tables S3 or S4 in the Supporting Information) under the constraint of a charge neutral system. Charge neutrality is essential, as the sum of all partial point charges of the system in an empirical force field typically has to be zero to avoid numerical instabilities due to long-range electrostatics described by an Ewald summation or similar. By limiting the partial charges of our force field to be charge neutral in total, the nonstoichiometry of the system is taken implicitly into account (see Methods). Finally, by adapting Bader charge redistributions in our empirical force field, we have succeeded in simulating thermodynamically stable $\mathrm{SCV}$ and DBT $\mathrm{Fe}_{3} \mathrm{O}_{4}(001)$ and $\mathrm{Fe}_{\text {tet1 }}{ }^{-}$ terminated $\mathrm{Fe}_{3} \mathrm{O}_{4}(111)$ surfaces at room temperature for several nanoseconds.

Formic Acid Adsorption on $\mathrm{Fe}_{3} \mathrm{O}_{4}$. Formic acid $(\mathrm{HCOOH})$ dissociates upon adsorption on magnetite to form formate $\left(\mathrm{HCOO}^{-}\right)$and a proton $\left(\mathrm{H}^{+}\right)$. In the case of magnetite (001) surfaces, it has been observed that the SCV reconstruction is lifted by formic acid adsorption already at low coverages. ${ }^{10,34}$ The focus is, in the following, hence on a DBT surface. After the dissociation reaction, $\mathrm{H}^{+}$binds to a nearby surface oxygen, and formate forms a bridging bidentate with two $\mathrm{Fe}_{\text {oct }}$ on the (001) surface. ${ }^{10,34}$ Formate either adsorbs next to an $\mathrm{Fe}_{\text {tet }}$ in the surface layer (called the "tet" adsorption site) or on a site between two $\mathrm{Fe}_{\text {tet }}$ ions (called the "int" adsorption site, cf. Figure 3). A favorable adsorption to "tet" sites was observed in DFT calculations and experiments for a fully formate-covered surface. ${ }^{34}$ The binding mode of formic acid on the $\mathrm{Fe}_{3} \mathrm{O}_{4}(111)$ surface is not yet studied in great detail. It is assumed that after initial adsorption to the closest oxygen, hydrogen migrates further to the second nearest neighbor oxygen, as previously observed for methanol dissociation on magnetite. ${ }^{29}$ A quasi-bidentate binding motif is established by binding of one of the formate oxygens to an $\mathrm{Fe}_{\text {tet1 }}$ and the other to the nearest surface hydroxyl group, eventually enabling a shuttling of hydrogen between them as observed on anatase $\mathrm{TiO}_{2}(101){ }^{77}$ Results of the Bader charge analysis after dissociation of formic acid and subsequent adsorption of formate on (001) and (111) magnetite surfaces are summarized in Tables S1 and S2. On both the (001) and (111) surfaces, iron atoms at the adsorption site lose electrons, while oxygens on the surface to which the dissociated hydrogen binds attract electrons. The Coulomb attraction between adsorbates and the surface is thus effectively increased, and ultimately, this configuration is energetically favored. On the magnetite (001) surface, the charge transfer upon dissociative adsorption of formic acid happens mainly in surface and subsurface layers. A fluctuating charge behavior in deeper surface regions is also observed on format-covered magnetite surfaces, as has been observed for bare surfaces.

Charges of our force field are adjusted from Bader charges in a similar way as before for bare surfaces. However, Bader charges on atoms associated with dissociative adsorption processes, that is, hydroxylated surface oxygen atoms and iron atoms connected to the adsorbed formate, differ significantly from the Bader charges on other atoms of the same kind in the same layer and a per-layer assignment of partial charges is thus not possible. Figure 3 summarizes, in a graphical illustration, resulting point charges of our force field which differ from bare surface charges. The definition of the angle between $\mathrm{Fe}-\mathrm{O}-\mathrm{H}$ is taken from CLAYFF and is defined for all interactions of a specific hydroxylated oxygen (i.e., three angles for every iron hydroxide).

In the following, results for different adsorption structures using our force field are compared against DFT calculations. However, accurate modeling of complete chemical reaction paths using an empirical force field is not straightforward, and real reaction paths are usually not fully reproducible. Nevertheless, applying an energy minimization to a fully covered, nonreconstructed magnetite (001) surface with formate at the "tet" or "int" site results in adsorption at "tet" being by $0.02 \mathrm{eV}$ more favorable than an adsorption to the "int" site. Quantitatively, this agrees reasonably well with energies obtained from DFT calculations, where the energy difference is $\Delta E_{\mathrm{ads}}=0.04 \mathrm{eV} .^{34}$ During thermalization of a half-covered surface with formate at $300 \mathrm{~K}$ for $1 \mathrm{~ns}$, no diffusion of formate between sites was observed. Diffusion barriers using the empirical force field for a single formate molecule on a halfcovered DBT surface were calculated using the nudged elastic band technique. ${ }^{78}$ Both directions require a significant amount of energy. Since the adsorption to an "int" site is energetically more favorable at low coverages (cf. Table S8), the diffusion from "int" to "tet" requires the crossing of an energetic barrier of $0.75 \mathrm{eV}$, while from "tet" to "int", it requires only $0.45 \mathrm{eV}$. However, these energies should be taken into consideration with caution, as the entire reaction path is not modeled. They are only meant to illustrate here that with our force field, it is unlikely that the molecule will diffuse on the surface.

Depending on the surface coverage, formate binds to the (111) magnetite surface in a quasi-bidentate or bidentate chelating configuration or mixture of both (cf. Figure 4). With the application of our empirical force field in the adsorption of 
a

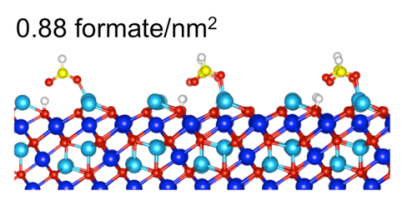

b 2.64 formate/nm²

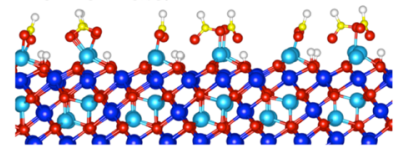

C 3.52 formate $/ \mathrm{nm}^{2}$

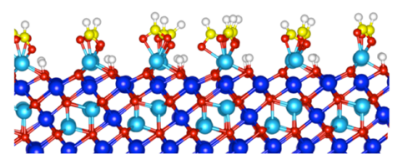

d 2.64 formate $/ \mathrm{nm}^{2}$

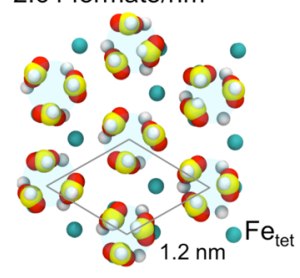

e 3.52 formate $/ \mathrm{nm}^{2}$

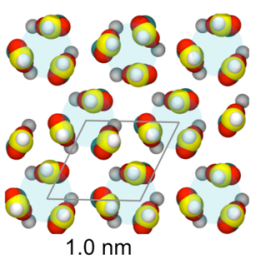

Figure 4. Adsorption of formate on an $\mathrm{Fe}_{\text {tet } 1}$-terminated (111) magnetite surface at different coverages results in a quasi-bidentate adsorption at 0.88 formate $/ \mathrm{nm}^{2}$, in (a), in a mixture of bidentate chelating and quasi-bidentate adsorption at 2.64 formate $/ \mathrm{nm}^{2}$, in (b), and in a pure bidentate chelating adsorption at 3.52 formate $/ \mathrm{nm}^{2}$, in (c). In (d,e), a top view of formate patterns observed on the (111) surface is shown. Color code: light blue $-\mathrm{Fe}_{\text {tet }}$, dark blue $-\mathrm{Fe}_{\text {oct }}$, redoxygen, yellow-carbon, and white-hydrogen.

molecular formic acid, a formic acid oxygen binds naturally to a tetrahedral iron surface atom, and the hydroxyl group of the formic acid binds to a nearby oxygen on the surface. Upon further dissociation, the former hydroxyl group oxygen establishes a hydrogen bond to a hydroxyl group on the surface (cf. Figure $4 \mathrm{a}$ ). Around 0.88 formate $/ \mathrm{nm}^{2}$, a quasi-bidentate adsorption is observed. By increasing the coverage to 2.64 formate $/ \mathrm{nm}^{2}$, a self-assembled, triangular-shaped arrangement of three formate molecules is observed (cf. Figure $4 b, d$ ). The triangular-shaped patterns have a periodicity of $1.2 \mathrm{~nm}$. In the case of a coverage of 3.52 formate $/ \mathrm{nm}^{2}$, all $\mathrm{Fe}_{\text {tet }}$ atoms on the (111) surface are occupied by formate, all second neighboring $\mathrm{O}_{1}$ atoms have formed surface hydroxyl groups, and another triangular-shaped assembly with a periodicity of $1.0 \mathrm{~nm}$ is observed. As a consequence, each formate is surrounded by 3 second next nearest hydroxyl groups (cf. Figure 4e). A forthcoming publication will present the initial experimental observation and characterization in combination with DFT calculations of such novel triangular-shaped superstructures on magnetite (111). ${ }^{53}$ However, our force field is capable of independently generating similar structures as those presented herein. Due to the weak bonding of the formate with the hydroxyl group, formate is able to dynamically rotate in steps of $120^{\circ}$ around its tetrahedral coordinated iron at room temperature on the (111) magnetite surface (see the second video in the Supporting Information). Formate is thus able to switch between different triangular-shaped clusters at room temperature at this coverage, leading to more irregular patterns on the magnetite surface, whereas at a coverage of 2.64 formate $/ \mathrm{nm}^{2}$, triangular clusters (Figure $4 \mathrm{~d}$ ) are always composed of the same three formate molecules the triangles are initially composed of. Reducing the temperature below $300 \mathrm{~K}$ stabilizes the configuration over the weak hydrogen bond. A reasonable geometrical agreement was additionally achieved between our empirical force field and structures obtained from DFT (cf. Figure S2 and ref 34).

In Figure 5, the validity of our new force field away from equilibrium adsorption structures is evaluated. Single-point
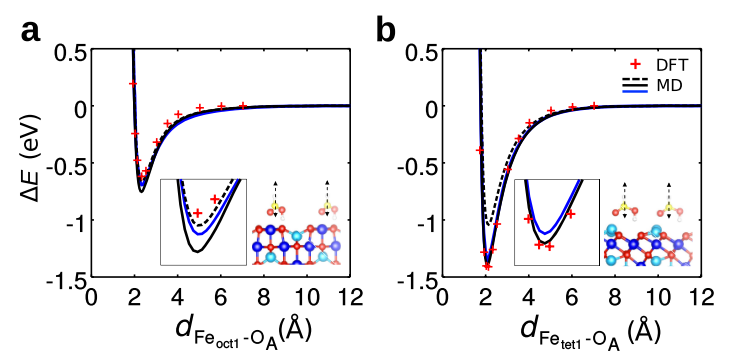

Figure 5. Potential energy-distance curves for formic acid ( $\mathrm{HCOOH})$ on magnetite surfaces using the new empirical force field along with reference DFT $(\mathrm{PBE}+U)$ data marked with red crosses. The energy difference per molecule $(\Delta E)$ is calculated with respect to the distance of the double-bonded oxygen of formic acid from the surface iron atom it is attached to. In $(a, b)$, formic acid is placed on an unreconstructed (001) and an $\mathrm{Fe}_{\text {tet } 1}$-terminated (111) magnetite surface, respectively. Black dashed lines correspond to partial point charges for iron atoms on bare surfaces (in (a), $+1.431 e$ for $\mathrm{Fe}_{\mathrm{oct} 1}$, and in (b), $+1.274 e$ for $\mathrm{Fe}_{\text {tet1 }}$ ). Black solid lines correspond to partial point charges of $\mathrm{Fe}_{\text {octl }}$ and $\mathrm{Fe}_{\text {tet1 }}$ in vicinity of a formate molecule (in (a), +1.621e for $\mathrm{Fe}_{\text {oct } 1}$; in (b), $+1.622 e$ for $\left.\mathrm{Fe}_{\text {tet } 1}\right)$. Blue lines are obtained using, for all atoms, partial point charges of the bulk magnetite crystal. However, this is a rather unphysical situation since formic acid dissociates immediately after adsorption to magnetite. Nevertheless, these comparisons are quite suitable for validating the force field here.

DFT energy reference calculations of a formic acid molecule fixed in its gas-phase structure and placed at varying distances to different magnetite surfaces were compared to results obtained with the force field. Three sets of parameters are compared in Figure 5: partial point charges corresponding (i) to bare surfaces, (ii) to formate-covered surfaces, and (iii) to bulk crystal partial charges. All parameter sets result in a good agreement with the DFT reference calculation for the DBT structure on the (001) magnetite surface, with bare partial charges performing the best. On the (111) surface, partial point charges for $\mathrm{Fe}_{\text {tet }}$ associated with formate adsorption correctly reproduce the DFT energies. This is explained by the similarity of molecularly adsorbed formic acid to a quasi-bound formate on the (111) magnetite surface. Bulk crystal partial charges are similar to the ones of a format-covered surface, which explains the almost equally good agreement.

Water Adsorption on $\mathrm{Fe}_{3} \mathrm{O}_{4}$. To test the more general applicability of our force field, water adsorption on magnetite surfaces is investigated. Bare magnetite surface charges are used here, with the exception that for the hydroxyl group $\mathrm{O}_{\mathrm{H}}$ and $\mathrm{H}_{\mathrm{O}}$ on the surface, original CLAYFF charges of $-0.95 e$ and $+0.425 e$, respectively, were used. A charge neutral slab is obtained by distributing the remaining residual charges on $\mathrm{Fe}_{\text {oct }}$ atoms connected to a hydroxyl group. A detailed summary of partial point charges used for water interfaces is found in Table S3.

Heats of immersion $E_{\text {him }}$ calculated from eq 4 and using our force field are calculated to be -598 and $-510 \mathrm{~mJ} / \mathrm{m}^{2}$ for a reconstructed SCV and unreconstructed DBT (001) magnetite surface, respectively. Both heats of immersion agree well with experimentally obtained values ranging between -519 and $-646 \mathrm{~mJ} / \mathrm{m}^{2}$ for magnetite, in general, that is, without specifying a certain surface. ${ }^{79}$

Previous theoretical studies based on DFT calculations have reported adsorption energies for up to four water molecules on 
the unreconstructed and reconstructed (001) magnetite surface. The most stable configuration of water on both (001) surfaces is a partially dissociated water dimer, ${ }^{12-14}$ where an undissociated water molecule and a surface hydroxyl group (from previously dissociated water) form a hydrogen-bonded $\mathrm{H}_{2} \mathrm{O} \cdots \mathrm{HO}$ pair (cf. Figure S2c,d). Partially dissociated water agglomerates on (001) magnetite surfaces have been reported to appear already at very low coverages. ${ }^{13}$ Due to the good availability of data for the (001) magnetite-water interface, molecular and partially dissociated water adsorptions on bare and hydroxylated surfaces are systematically investigated using our force field and compared to previous DFT calculations. The length of the hydrogen bond between $\mathrm{H}_{2} \mathrm{O}$ and $\mathrm{HO}$ is somewhat shorter $(\sim 1.60 \AA$ on SCV and DBT) than the one between two nondissociated water molecules $(\sim 1.90 \AA \AA$ on SCV and DBT). Furthermore, in a mixed-mode adsorption, the bond length between $\mathrm{Fe}_{\text {oct } 1}$ and water oxygen $\mathrm{O}_{\mathrm{W}}$ is slightly shorter than that of a molecular water dimer for both SCV and DBT surface terminations. The shortening of the bond lengths could conceivably be attributed to the cooperativity effect. ${ }^{13,80}$ Overall, distances summarized in Figure S2c,d correspond to distances from DFT calculations remarkably well (cf. refs 12 and 13).

Various calculated adsorption energies for different water coverages on magnetite are summarized in Table 2 and divided

Table 2. Comparison of Molecular Adsorption Energies $E_{\text {ads }}$ and Mixed-Mode Adsorption Energies $E_{\text {ads }}^{*}$ (See Eqs 5 and 6 in the Methods Section) for Water Clusters on the $\mathrm{Fe}_{3} \mathrm{O}_{4}(001)$ Surfaces Using Our Force Field with DFT Values Found in the Literature ${ }^{a}$

\begin{tabular}{cccccc} 
& \multicolumn{2}{c}{$\mathrm{SCV}$} & & \multicolumn{2}{c}{$\mathrm{DBT}$} \\
\cline { 2 - 3 } \cline { 5 - 6 } molecular adsorption & $E_{\text {ads }}(\mathrm{MD})$ & $E_{\text {ads }}(\mathrm{DFT})$ & & $E_{\text {ads }}(\mathrm{MD})$ & $E_{\text {ads }}(\mathrm{DFT})$ \\
monomer $\left(\mathrm{H}_{2} \mathrm{O}\right)$ & -0.55 & $-0.94^{c}$, & & -0.62 & $-0.39^{b}$, \\
& & $-0.64^{d}$ & & & $-1.10^{c}$ \\
dimer $\left(\mathrm{H}_{2} \mathrm{O}\right)_{2}$ & -0.62 & $-0.66^{d}$ & & -0.64 & $-0.47^{b}$ \\
trimer $\left(\mathrm{H}_{2} \mathrm{O}\right)_{3}$ & -0.64 & $\mathrm{n} / \mathrm{a}$ & & -0.64 & $\mathrm{n} / \mathrm{a}$ \\
tetramer $\left(\mathrm{H}_{2} \mathrm{O}\right)_{4}$ & -0.68 & $-0.94^{c}$ & & -0.70 & $-0.51^{b}$, \\
& & & & $-1.05^{c}$ \\
mixed adsorption & $E_{\text {ads }}^{*}(\mathrm{MD})$ & $E_{\text {ads }}(\mathrm{DFT})$ & $E_{\text {ads }}^{*}(\mathrm{MD})$ & $E_{\text {ads }}(\mathrm{DFT})$ \\
$\left(\mathrm{H}_{2} \mathrm{O}\right) \cdots \mathrm{HO}$ & -0.93 & $-0.92^{d}$ & -1.01 & $-0.94^{b}$ \\
$\left(\mathrm{H}_{2} \mathrm{O}\right)_{2} \cdots \mathrm{HO}$ & -0.83 & $-0.88^{d}$ & -0.86 & $\mathrm{n} / \mathrm{a}$ \\
$\left(\mathrm{H}_{2} \mathrm{O}\right)_{2} \cdots(\mathrm{HO})_{2}$ & -0.72 & $-0.98^{c}$ & -1.12 & $-0.82^{b},-1.42^{c}$
\end{tabular}

${ }^{a}$ Energies are given in eV. ${ }^{b}$ Taken from ref $12 .{ }^{c}$ Taken from ref 14. $d_{\text {Taken from ref } 13 .}$

into molecular and mixed adsorption. However, comparisons between energies for different water coverages are typically not directly accessible, particularly not between molecular and partially dissociated water clusters, since the dissociation energy of water in empirical force fields is typically not exactly reproducible.

Although a comparison between mixed and molecular adsorption using an empirical force field should thus be treated with caution, our force field is quite reliable in predicting relative energy differences compared to DFT calculations. Energy differences between mixed and molecular adsorption of water on SCV- and DBT-terminated magnetite (001) surfaces using the empirical force field are, in most cases, similar to DFT calculations, indicating at least some agreement between DFT and the empirical force field regarding the dissociation energy of water. On both DBT and SCV surface structures, the energetically more stable partially dissociated water dimer is preferred over the molecular water dimer. With increasing coverage, mixed adsorption modes on either reconstructed or unreconstructed (001) magnetite surfaces are found to be more favorable than molecular adsorptions when compared directly $\left(\mathrm{H}_{2} \mathrm{O} \cdots \mathrm{HO}\right.$ vs $\left(\mathrm{H}_{2} \mathrm{O}\right)_{2}$, etc. $)$. The lowest-energy configuration of water on an unreconstructed (001) magnetite surface using our empirical force field is a partially dissociated water tetramer. Contrary to results on the unreconstructed (001) surface, on the reconstructed (001) surface, an increasing water coverage reduces the adsorption energy per water molecule in a mixed adsorption.

Due to the energetically more favorable partial dissociation of water, water networks are further investigated by successively increasing the water coverage starting from a partially dissociated water tetramer, as shown in Figure 6. At full monolayer water coverage, all four $\mathrm{Fe}_{\text {octl }}$ sites in each $(\sqrt{2} \times \sqrt{2}) R 45^{\circ}$ unit cell are occupied by two $\mathrm{H}_{2} \mathrm{O}$ and two $\mathrm{OH}$ groups (cf. Figure 6a).

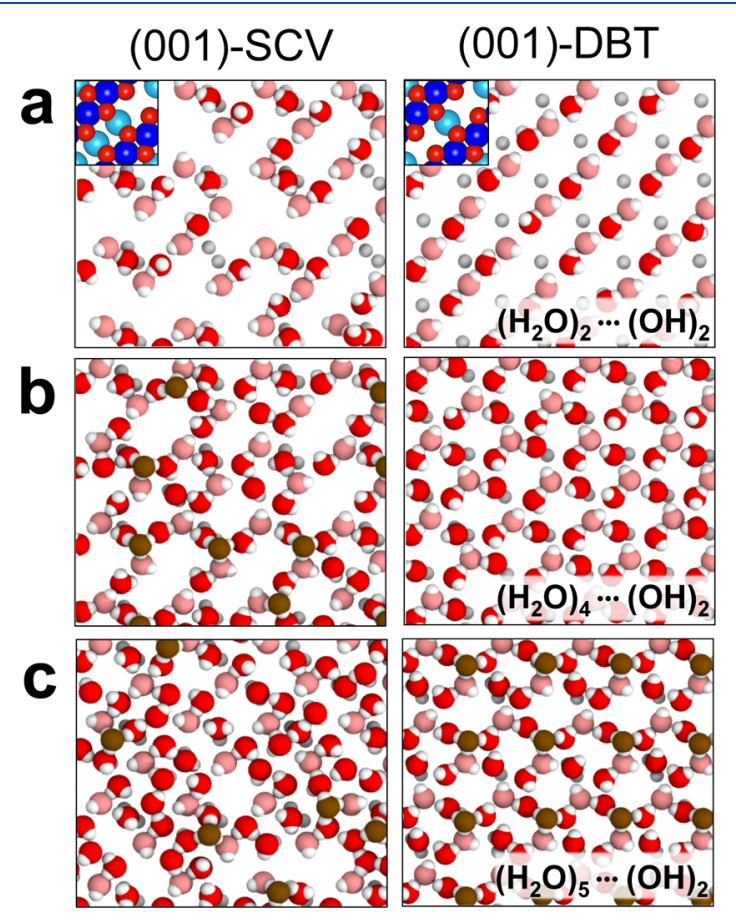

Figure 6. Water adsorption on SCV (left) and DBT (right) at $300 \mathrm{~K}$. Top views of water patterns obtained for different coverages: (a) $\left(\mathrm{H}_{2} \mathrm{O}\right)_{2} \cdots(\mathrm{OH})_{2},(\mathrm{~b})\left(\mathrm{H}_{2} \mathrm{O}\right)_{4} \cdots(\mathrm{OH})_{2}$, and $(\mathrm{c})\left(\mathrm{H}_{2} \mathrm{O}\right)_{5} \cdots(\mathrm{OH})_{2}$ per unit cell. Inset of $(\mathrm{a})$, the $(\sqrt{2} \times \sqrt{2}) R 45^{\circ}$ unit cells are shown. In molecular water, oxygen is red and hydrogen is white. For dissociated water, the oxygen atoms of $\mathrm{OH}$ groups are highlighted in pink. Following the partially dissociated tetramer, the first layer is covered with a successive layer that consists of the water molecules depicted in brown.

As a result of the full coverage on an SCV surface termination, surface hydroxyl group hydrogens in the two $\mathrm{Fe}_{\text {octl }}$ rows face each other directly (see Figure 6a). Due to repulsive interactions between opposing hydroxyl groups, eventually a partially dissociated tetramer on a reconstructed SCV (001) magnetite surface $(-0.72 \mathrm{eV})$ is energetically more unfavorable than a dimer $(-0.93 \mathrm{eV})$ and a trimer $(-0.83 \mathrm{eV})$ configuration. However, tetramer water patterns, observed in Figure 6a, on an SCV surface termination agree with nc-AFM results of Meier et 
al., ${ }^{13}$ which they have observed at a coverage of $2.5 \pm 0.5 \mathrm{H}_{2} \mathrm{O}$ molecules per unit cell. In this configuration, some water molecules inside a tetramer configuration on SCV are bridging $\mathrm{Fe}_{\text {oct } 1}$ rows at which surface hydroxyl groups provide more hydrogen bonds.

At a coverage of $\left(\mathrm{H}_{2} \mathrm{O}\right)_{5} \cdots(\mathrm{OH})_{2}$, a similar but much more ordered water bridging structure is formed on a DBT surface termination. Water molecules arrange along the $\mathrm{Fe}_{\text {oct1 }}$ row, creating a regular pattern of hydrogen bonds. It is assumed that a DBT surface structure provides a more stable hydrogen-bonded water network than an SCV surface structure and that hydrogen on the surface, moreover, plays a key role in the formation of adsorption structures.

\section{CONCLUSIONS}

In this work, results for a particularly interesting interface between magnetite and a prototypic carboxylic acid (formic acid and its dissociation product formate) related to crystal growth and water interfaces are presented based on a newly parameterized force field potential. An existing force field for ferric oxyhydroxide ${ }^{63,66}$ was extended for magnetite in this work by adapting partial point charges in an empirical force field from a DFT-based Bader charge analysis and, in addition, ensuring compatibility with standard biomolecular force fields by careful parameterization against a widely used force field for small organic molecules $\left(\mathrm{GAFF}^{68}\right)$. Two prominent surface terminations of $\mathrm{Fe}_{3} \mathrm{O}_{4}(001)$, an SCV and a DBT, are stable at $300 \mathrm{~K}$ in vacuum using our force field. It was observed that the charge redistribution after cleavage of the bulk crystal is eventually a driving force of the surface stability. In particular, octahedrally coordinated irons are more positively charged on the surface.

Comparison to DFT calculations of formic acid adsorption on magnetite (001) surfaces proves that our empirical force field parameterization is robust and accurate. A preferential adsorption of formate to the "tet" sites on the (001) magnetite surface was observed when fully covered. At half formate coverage, an adsorption to "int" sites is energetically more favorable than to "tet" sites. It should be noted furthermore that a formic acid molecule adsorbing to a bare (001) magnetite surface can encounter an SCV surface termination, where a favorable binding to "int" sites was observed also in DFT calculations. Both eventually explain the significant amount of formic acid at "int" sites on an almost completely covered magnetite (001) surface observed in surface X-ray diffraction (XRD) experiments. ${ }^{34}$ On the $\mathrm{Fe}_{\text {tet } 1}$-terminated (111) magnetite surface, a hydrogen bond mediating arrangement of formic acid results in two different triangular patterns of formate whose (dynamic) structures depend on both temperature and coverage. Based on formic acid adsorption and dissociation, the archetypal dissociation of more complex fatty acids on magnetite using the presented force field is currently being investigated by comparing results from the empirical force field with experimental Fourier transform infrared and XRD signals and will be published in a follow-up study. Oleic acid, for example, plays a prominent role in the growth of magnetite nanoparticles from solution ${ }^{16}$ and in the extraordinary mechanical strength of the nanocomposites of Dreyer et al. ${ }^{4}$

The interface between a (001) magnetite surface and water ranging from a few water molecules per unit cell up to a full liquid layer was furthermore investigated. In ref 37, several difficulties in the application of an empirical magnetite force field arose that could not be solved completely. For example, the interaction between $\mathrm{Fe}$ and water was overestimated, and thus, the distance between $\mathrm{Fe}$ and $\mathrm{OH}_{2}$ was underestimated. Another reported problem was the poor transferability of the LJ 9-6 cross terms of the COMPASS-based water model. It is also important to note that the fixation of the atoms within the hydroxylated $\mathrm{Fe}_{3} \mathrm{O}_{4}(001)$ slab herein resulted in overestimated interatomic distances and underestimated interaction energies. In our approach, however, the description of hydroxylated magnetite is based on modified CLAYFF ${ }^{63}$ parameters that reproduce well both structural observations for bulk magnetite and known surfaces from DFT calculations and experiments. The CLAYFF parameters are usually also compatible with common water models and established biomolecular force fields, ${ }^{64}$ on which we have put an additional focus here to ensure compatibility. Overall, this gives this approach a significant advantage over previous approaches, since it allows us to accurately model the entire system without imposing any restrictions on the magnetite atoms, as is the case when modeling magnetite substrates with frozen atoms. Thus, in contrast to previous studies of Liu et al. ${ }^{37}$ and Rustad ${ }^{81}$ using an empirical force field as well, atomic distances between magnetite and water are in good agreement with DFT calculations ${ }^{12,13}$ using our new empirical force field. Upon examination of the stability of molecular and dissociatively bonded water clusters on SCV- and DBT-terminated magnetite (001) surfaces, an ordered water monolayer forming a network connected by hydrogen bonds is thermodynamically more stable on the DBT structure at room temperature than the more unordered arrangement of water observed on the reconstructed SCV surface covered with the same amount of water. Calculated heats of immersion using our empirical force field are in good agreement with experimental values found in the literature. However, despite recent advances regarding the understanding of the behavior of water on (111) magnetite surfaces, ${ }^{27}$ there are still fundamental issues to be clarified such as the stability of ordered structures and ice nucleation. Such a detailed analysis was not performed for this surface, although our force field demonstrates great potential. Since we only assess the performance of our force field on three well-defined magnetite surfaces (i.e., (001)-DBT, (001)-SCV, and (111)- $-\mathrm{Fe}_{\text {tet } 1}$ ) and under well-defined conditions (i.e., room temperature well above the Verwey transition, atmospheric pressure, and, apart from a liquid layer of water, only specific adsorption motifs of small organic molecules), there are some important points that must be carefully evaluated before using this force field. For this reason, great caution is still needed when using this potential to model complex magnetite structures with surface defects or interaction with other organic molecules. As surface complexity increases, the simulations should be validated by a suitable comparison with experimental results and DFT calculations. Nevertheless, the approach presented here shows a possible way to achieve this.

In summary, our approach based on an empirical force field with point charges adapted from a Bader charge analysis provides a reliable method for modeling magnetite-organic interfaces. Apart from enabling simulations of magnetite in aqueous environments, our force field has the potential to be used for a deeper analysis of organically coated magnetite surfaces, for example, the self-assembly of ligands or mechanical properties of organically functionalized magnetite nanoparticles, and is easily extended for use with other ligands such as oleic or phosphonic acids. Furthermore, our force field is particularly useful in order to understand atomistic mechanisms of the crystallization by colloidal assembly ${ }^{82}$ and shape-forming criteria of magnetite nanoparticles. ${ }^{56}$ In both cases, our force 
field is readily applicable in crystal growth simulations of magnetite, for example, using a Kawska-Zahn approach. ${ }^{83}$ The possibility of generating positive or negative surface charges on the magnetite nanoparticles by altering the organic linker molecules might be important in a polymer-assisted colloidal crystallization as well. ${ }^{84}$

\section{ASSOCIATED CONTENT}

\section{SI Supporting Information}

The Supporting Information is available free of charge at https://pubs.acs.org/doi/10.1021/acs.jpcc.0c10338.

Bader charges from DFT calculations, partial point charges used in the empirical force field, structural details of magnetite interfaces, and energetic details of magnetite (001) interfaces to formic acid/formate (PDF)

Diffusion of iron atoms and a nonphysical and rather unstructured surface termination when employing bulk partial charges (MP4)

Dynamic rotation in steps of $120^{\circ}$ of formate (MP4)

\section{AUTHOR INFORMATION}

\section{Corresponding Author}

Robert H. Meißner - Institute of Polymers and Composites, Hamburg University of Technology, 21073 Hamburg, Germany; Institute of Materials Research, MagIC-Magnesium Innovation Centre, Helmholtz-Zentrum Geesthacht, 21502 Geesthacht, Germany; (1) orcid.org/0000-0003-1926-114X; Phone: +49 4042878 2580; Email: robert.meissner@ tuhh.de

\section{Authors}

Mine Konuk - Institute of Polymers and Composites, Hamburg University of Technology, 21073 Hamburg, Germany

Kai Sellschopp - Institute of Advanced Ceramics, Hamburg University of Technology, 21073 Hamburg, Germany; (1) orcid.org/0000-0002-0003-2075

Gregor B. Vonbun-Feldbauer - Institute of Advanced Ceramics, Hamburg University of Technology, 21073 Hamburg, Germany; 이이이.org/0000-0002-9327-0450

Complete contact information is available at: https://pubs.acs.org/10.1021/acs.jpcc.0c10338

\section{Notes}

The authors declare no competing financial interest.

\section{ACKNOWLEDGMENTS}

We want to thank Lucio Colombi Ciacchi for the fruitful and constructive discussion on the manuscript, Wernfried MayrSchmölzer for helping in improving the settings of the DFT calculations, and Tim Würger for support to calculate RESP charges. The project was funded by the Deutsche Forschungsgemeinschaft (DFG, German Research Foundation)-Projektnummer 192346071-SFB 986, and-Projektnummer $390794421-$ GRK 2462.

\section{REFERENCES}

(1) Wegst, U. G. K.; Bai, H.; Saiz, E.; Tomsia, A. P.; Ritchie, R. O. Bioinspired structural materials. Nat. Mater. 2014, 14, 23-36.

(2) Veedu, V. P.; Cao, A.; Li, X.; Ma, K.; Soldano, C.; Kar, S.; Ajayan, P. M.; Ghasemi-Nejhad, M. N. Multifunctional composites using reinforced laminae with carbon-nanotube forests. Nat. Mater. 2006, 5, $457-462$.
(3) Dimas, L. S.; Bratzel, G. H.; Eylon, I.; Buehler, M. J. Tough Composites Inspired by Mineralized Natural Materials: Computation, 3D printing, and Testing. Adv. Funct. Mater. 2013, 23, 4629-4638.

(4) Dreyer, A.; Feld, A.; Kornowski, A.; Yilmaz, E. D.; Noei, H.; Meyer, A.; Krekeler, T.; Jiao, C.; Stierle, A.; Abetz, V.; et al. Organically linked iron oxide nanoparticle supercrystals with exceptional isotropic mechanical properties. Nat. Mater. 2016, 15, 522-528.

(5) Giuntini, D.; Torresani, E.; Chan, K. T.; Blankenburg, M.; Saviot, L.; Bor, B.; Domènech, B.; Shachar, M.; Müller, M.; Olevsky, E. A.; et al. Iron oxide-based nanostructured ceramics with tailored magnetic and mechanical properties: development of mechanically robust, bulk superparamagnetic materials. Nanoscale Adv. 2019, 1, 3139-3150.

(6) Domènech, B.; Kampferbeck, M.; Larsson, E.; Krekeler, T.; Bor, B.; Giuntini, D.; Blankenburg, M.; Ritter, M.; Müller, M.; Vossmeyer, T.; et al. Hierarchical supercrystalline nanocomposites through the self-assembly of organically-modified ceramic nanoparticles. Sci. Rep. 2019, 9, 3435.

(7) Patitsa, M.; Karathanou, K.; Kanaki, Z.; Tzioga, L.; Pippa, N.; Demetzos, C.; Verganelakis, D. A.; Cournia, Z.; Klinakis, A. Magnetic nanoparticles coated with polyarabic acid demonstrate enhanced drug delivery and imaging properties for cancer theranostic applications. Sci. Rep. 2017, 7, 775.

(8) Sarcletti, M.; Vivod, D.; Luchs, T.; Rejek, T.; Portilla, L.; Müller, L.; Dietrich, H.; Hirsch, A.; Zahn, D.; Halik, M. Superoleophilic Magnetic Iron Oxide Nanoparticles for Effective Hydrocarbon Removal from Water. Adv. Funct. Mater. 2019, 29, 1805742.

(9) Arndt, B.; Bliem, R.; Gamba, O.; van der Hoeven, J. E. S.; Noei, H.; Diebold, U.; Parkinson, G. S.; Stierle, A. Atomic structure and stability of magnetite $\mathrm{Fe}_{3} \mathrm{O}_{4}(001)$ : An X-ray view. Surf. Sci. 2016, 653, 76-81.

(10) Gamba, O.; Noei, H.; Pavelec, J.; Bliem, R.; Schmid, M.; Diebold, U.; Stierle, A.; Parkinson, G. S. Adsorption of Formic Acid on the $\mathrm{Fe}_{3} \mathrm{O}_{4}(001)$ Surface. J. Phys. Chem. C 2015, 119, 20459-20465.

(11) Hosseini nasr, A.-s.; Akbarzadeh, H.; Tayebee, R. Adsorption mechanism of different acyclovir concentrations on $1-2 \mathrm{~nm}$ sized magnetite nanoparticles: A molecular dynamics study. J. Mol. Liq. 2018, 254, 64-69.

(12) Mulakaluri, N.; Pentcheva, R.; Scheffler, M. Coverage-Dependent Adsorption Mode of Water on $\mathrm{Fe}_{3} \mathrm{O}_{4}(001)$ : Insights from First Principles Calculations. J. Phys. Chem. C 2010, 114, 11148-11156.

(13) Meier, M.; Hulva, J.; Jakub, Z.; Pavelec, J.; Setvin, M.; Bliem, R.; Schmid, M.; Diebold, U.; Franchini, C.; Parkinson, G. S. Water agglomerates on $\mathrm{Fe}_{3} \mathrm{O}_{4}(001)$. Proc. Natl. Acad. Sci. U.S.A. 2018, 115, E5642-E5650.

(14) Liu, H.; Di Valentin, C. Bulk-terminated or reconstructed $\mathrm{Fe}_{3} \mathrm{O}_{4}(001)$ surface: water makes a difference. Nanoscale 2018, 10, 11021-11027.

(15) Rawlings, A. E.; Bramble, J. P.; Tang, A. A. S.; Somner, L. A.; Monnington, A. E.; Cooke, D. J.; McPherson, M. J.; Tomlinson, D. C.; Staniland, S. S. Phage display selected magnetite interacting Adhirons for shape controlled nanoparticle synthesis. Chem. Sci. 2015, 6, 55865594.

(16) Feld, A.; Weimer, A.; Kornowski, A.; Winckelmans, N.; Merkl, J.P.; Kloust, H.; Zierold, R.; Schmidtke, C.; Schotten, T.; Riedner, M.; et al. Chemistry of Shape-Controlled Iron Oxide Nanocrystal Formation. ACS Nano 2019, 13, 152-162.

(17) Parkinson, G. S. Iron oxide surfaces. Surf. Sci. Rep. 2016, 71, $272-$ 365.

(18) Pentcheva, R.; Wendler, F.; Meyerheim, H. L.; Moritz, W.; Jedrecy, N.; Scheffler, M. Jahn-Teller Stabilization of a "Polar" Metal Oxide Surface: $\mathrm{Fe}_{3} \mathrm{O}_{4}(001)$. Phys. Rev. Lett. 2005, 94, 126101.

(19) Bliem, R.; McDermott, E.; Ferstl, P.; Setvin, M.; Gamba, O.; Pavelec, J.; Schneider, M. A.; Schmid, M.; Diebold, U.; Blaha, P.; et al. Subsurface cation vacancy stabilization of the magnetite (001) surface. Science 2014, 346, 1215-1218.

(20) Li, X.; Paier, J. Adsorption of Water on the $\mathrm{Fe}_{3} \mathrm{O}_{4}(111)$ Surface: Structures, Stabilities, and Vibrational Properties Studied by Density Functional Theory. J. Phys. Chem. C 2016, 120, 1056-1065.

(21) Noh, J.; Osman, O. I.; Aziz, S. G.; Winget, P.; Brédas, J.-L. Magnetite $\mathrm{Fe}_{3} \mathrm{O}_{4}(111)$ Surfaces: Impact of Defects on Structure, 
Stability, and Electronic Properties. Chem. Mater. 2015, 27, 58565867.

(22) Santos-Carballal, D.; Roldan, A.; Grau-Crespo, R.; de Leeuw, N. $\mathrm{H}$. A DFT study of the structures, stabilities and redox behaviour of the major surfaces of magnetite $\mathrm{Fe}_{3} \mathrm{O}_{4}$. Phys. Chem. Chem. Phys. 2014, 16, 21082-21097.

(23) Yu, X.; Huo, C.-F.; Li, Y.-W.; Wang, J.; Jiao, H. $\mathrm{Fe}_{3} \mathrm{O}_{4}$ surface electronic structures and stability from GGA+U. Surf. Sci. 2012, 606, 872-879.

(24) Shaikhutdinov, S. K.; Ritter, M.; Wang, X.-G.; Over, H.; Weiss, W. Defect structures on epitaxial $\mathrm{Fe}_{3} \mathrm{O}_{4}(111)$ films. Phys. Rev. B: Condens. Matter Mater. Phys. 1999, 60, 11062-11069.

(25) Li, X.; Paier, J.; Sauer, J.; Mirabella, F.; Zaki, E.; Ivars-Barceló, F.; Shaikhutdinov, S.; Freund, H.-J. Surface Termination of $\mathrm{Fe}_{3} \mathrm{O}_{4}(111)$ Films Studied by CO Adsorption Revisited. J. Phys. Chem. B 2018, 122, 527-533.

(26) Asakawa, K.; Miura, Y.; Nagatsuka, N.; Takeyasu, K.; Matsumoto, M.; Fukutani, K. Electronic and spin structure of O- and $\mathrm{H}$-adsorbed $\mathrm{Fe}_{3} \mathrm{O}_{4}(111)$ surfaces. Phys. Rev. B 2019, 99, 085442.

(27) Zaki, E.; Jakub, Z.; Mirabella, F.; Parkinson, G. S.; Shaikhutdinov, S.; Freund, H.-J. Water Ordering on the Magnetite $\mathrm{Fe}_{3} \mathrm{O}_{4}$ Surfaces. J. Phys. Chem. Lett. 2019, 10, 2487-2492.

(28) Xu, F.; Chen, W.; Walenta, C. A.; O'Connor, C. R.; Friend, C. M. Dual Lewis site creation for activation of methanol on $\mathrm{Fe}_{3} \mathrm{O}_{4}(111)$ thin films. Chem. Sci. 2020, 11, 2448-2454.

(29) Li, X.; Paier, J. Partial Oxidation of Methanol on the $\mathrm{Fe}_{3} \mathrm{O}_{4}(111)$ Surface Studied by Density Functional Theory. J. Phys. Chem. C 2019, 123, 8429-8438.

(30) Pavelec, J.; Hulva, J.; Halwidl, D.; Bliem, R.; Gamba, O.; Jakub, Z.; Brunbauer, F.; Schmid, M.; Diebold, U.; Parkinson, G. S. A multitechnique study of $\mathrm{CO} 2$ adsorption on $\mathrm{Fe}_{3} \mathrm{O}_{4}$ magnetite. J. Chem. Phys. 2017, 146, 014701.

(31) Mirabella, F.; Zaki, E.; Ivars-Barcelo, F.; Schauermann, S.; Shaikhutdinov, S.; Freund, H.-J. CO2 Adsorption on Magnetite $\mathrm{Fe}_{3} \mathrm{O}_{4}(111)$. J. Phys. Chem. C 2018, 122, 27433-27441.

(32) Wojas, J.; Kwiatek, N.; Wilgocka-Slęzak, D.; Madej, E.; Korecki, J.; Spiridis, N. CO adsorption on $\mathrm{Fe}_{3} \mathrm{O}_{4}(111)$ with regular and biphase terminations. Appl. Surf. Sci. 2020, 507, 145069.

(33) Parkinson, G. S.; Mulakaluri, N.; Losovyj, Y.; Jacobson, P.; Pentcheva, R.; Diebold, U. Semiconductor-half metal transition at the $\mathrm{Fe}_{3} \mathrm{O}_{4}(001)$ surface upon hydrogen adsorption. Phys. Rev. B: Condens. Matter Mater. Phys. 2010, 82, 125413.

(34) Arndt, B.; Sellschopp, K.; Creutzburg, M.; Grånäs, E.; Krausert, K.; Vonk, V.; Müller, S.; Noei, H.; Feldbauer, G. B. V.; Stierle, A. Carboxylic acid induced near-surface restructuring of a magnetite surface. Commun. Chem. 2019, 2, 92.

(35) Kraushofer, F.; Mirabella, F.; Xu, J.; Pavelec, J.; Balajka, J.; Müllner, M.; Resch, N.; Jakub, Z.; Hulva, J.; Meier, M.; et al. Self-limited growth of an oxyhydroxide phase at the $\mathrm{Fe}_{3} \mathrm{O}_{4}(001)$ surface in liquid and ambient pressure water. J. Chem. Phys. 2019, 151, 154702.

(36) Arndt, B.; Creutzburg, M.; GrÅnäs, E.; Volkov, S.; Krausert, K.; Vlad, A.; Noei, H.; Stierle, A. Water and Atomic Hydrogen Adsorption on Magnetite (001). J. Phys. Chem. C 2019, 123, 26662-26672.

(37) Liu, H.; Bianchetti, E.; Siani, P.; Di Valentin, C. Insight into the interface between $\mathrm{Fe}_{3} \mathrm{O}_{4}(001)$ surface and water overlayers through multiscale molecular dynamics simulations. J. Chem. Phys. 2020, 152, 124711.

(38) Larrucea, J.; Lid, S.; Colombi Ciacchi, L. Parametrization of a classical force field for iron oxyhydroxide/water interfaces based on Density Functional Theory calculations. Comput. Mater. Sci. 2014, 92, 343-352.

(39) Tromp, S.; Joly, L.; Cobian, M.; Fillot, N. Chemical Physics at Interfaces within a Refrigerant-Lubricated Contact: From Electronic Structure to Large-Scale Molecular Dynamics Simulations. J. Phys. Chem. C 2018, 122, 5420-5429.

(40) Latorre, C. A.; Ewen, J. P.; Gattinoni, C.; Dini, D. Simulating Surfactant-Iron Oxide Interfaces: From Density Functional Theory to Molecular Dynamics. J. Phys. Chem. B 2019, 123, 6870-6881.
(41) Kundu, T. K.; Rao, K. H.; Parker, S. C. Atomistic simulation studies of magnetite surface structures and adsorption behavior in the presence of molecular and dissociated water and formic acid. J. Colloid Interface Sci. 2006, 295, 364-373.

(42) Martin, L.; Bilek, M. M.; Weiss, A. S.; Kuyucak, S. Force fields for simulating the interaction of surfaces with biological molecules. Interface Focus 2016, 6, 20150045.

(43) Cornell, W. D.; Cieplak, P.; Bayly, C. I.; Kollman, P. A. Application of RESP Charges To Calculate Conformational Energies, Hydrogen Bond Energies, and Free Energies of Solvation. J. Am. Chem. Soc. 1993, 115, 9620-9631.

(44) Bader, R.; Bader, R. Atoms in Molecules: A Quantum Theory; International Series of Monographs on Chemistry; Clarendon Press, 1990.

(45) Henkelman, G.; Arnaldsson, A.; Jónsson, H. A fast and robust algorithm for Bader decomposition of charge density. Comput. Mater. Sci. 2006, 36, 354-360.

(46) Kresse, G.; Furthmüller, J. Efficient Iterative Schemes for Ab Initio Total-Energy Calculations Using a Plane-Wave Basis Set. Phys. Rev. B: Condens. Matter Mater. Phys. 1996, 54, 11169-11186.

(47) Kresse, G.; Furthmüller, J. Efficiency of Ab-Initio Total Energy Calculations for Metals and Semiconductors Using a Plane-Wave Basis Set. Comput. Mater. Sci. 1996, 6, 15-50.

(48) Blöchl, P. E. Projector Augmented-Wave Method. Phys. Rev. B: Condens. Matter Mater. Phys. 1994, 50, 17953-17979.

(49) Perdew, J. P.; Burke, K.; Ernzerhof, M. Generalized Gradient Approximation Made Simple. Phys. Rev. Lett. 1996, 77, 3865-3868.

(50) Dudarev, S. L.; Botton, G. A.; Savrasov, S. Y.; Humphreys, C. J.; Sutton, A. P. Electron-Energy-Loss Spectra and the Structural Stability of Nickel Oxide: An LSDA+U Study. Phys. Rev. B: Condens. Matter Mater. Phys. 1998, 57, 1505-1509.

(51) Guo, H.; Barnard, A. S. Modeling the Iron Oxides and Oxyhydroxides for the Prediction of Environmentally Sensitive Phase Transformations. Phys. Rev. B: Condens. Matter Mater. Phys. 2011, 83, 094112.

(52) Meng, Y.; Liu, X.-W.; Huo, C.-F.; Guo, W.-P.; Cao, D.-B.; Peng, Q.; Dearden, A.; Gonze, X.; Yang, Y.; Wang, J.; et al. When Density Functional Approximations Meet Iron Oxides. J. Chem. Theory Comput. 2016, 12, 5132-5144.

(53) Creutzburg, M.; Sellschopp, K.; Tober, S.; Grånäs, E.; Vonk, V.; Mayr-Schmölzer, W.; Müller, S.; Noei, H.; Vonbun-Feldbauer, G.; Stierle, A. Heterogeneous Adsorption and Local Ordering of Formate on a Magnetite Surface. ChemRxiv 2021, DOI: 10.26434/chemrxiv.13606685.v1.

(54) Walsh, A.; Sokol, A. A.; Buckeridge, J.; Scanlon, D. O.; Catlow, C. R. A. Oxidation states and ionicity. Nat. Mater. 2018, 17, 958-964.

(55) Butenuth, A.; Moras, G.; Schneider, J.; Koleini, M.; Köppen, S.; Meißner, R.; Wright, L. B.; Walsh, T. R.; Ciacchi, L. C. Ab initio derived force-field parameters for molecular dynamics simulations of deprotonated amorphous-SiO2/water interfaces. Phys. Status Solidi B 2012, 249, 292-305.

(56) Liu, H.; Di Valentin, C. Shaping Magnetite Nanoparticles from First Principles. Phys. Rev. Lett. 2019, 123, 186101.

(57) Lundborg, M.; Lindahl, E. Automatic GROMACS Topology Generation and Comparisons of Force Fields for Solvation Free Energy Calculations. J. Phys. Chem. B 2015, 119, 810-823.

(58) Plimpton, S. Fast Parallel Algorithms for Short-Range Molecular Dynamics. J. Comput. Phys. 1995, 117, 1-19.

(59) Maier, J. A.; Martinez, C.; Kasavajhala, K.; Wickstrom, L.; Hauser, K. E.; Simmerling, C. ff14SB: Improving the Accuracy of Protein Side Chain and Backbone Parameters from ff99SB. J. Chem. Theory Comput. 2015, 11, 3696-3713.

(60) Brooks, B. R.; Brooks, C. L., III; Mackerell, A. D., Jr.; Nilsson, L.; Petrella, R. J.; Roux, B.; Won, Y.; Archontis, G.; Bartels, C.; Boresch, S.; et al. CHARMM: The biomolecular simulation program. J. Comput. Chem. 2009, 30, 1545-1614.

(61) Heinz, H.; Lin, T.-J.; Kishore Mishra, R.; Emami, F. S. Thermodynamically Consistent Force Fields for the Assembly of 
Inorganic, Organic, and Biological Nanostructures: The INTERFACE Force Field. Langmuir 2013, 29, 1754-1765.

(62) Cruz-Chu, E. R.; Aksimentiev, A.; Schulten, K. Water-Silica Force Field for Simulating Nanodevices. J. Phys. Chem. B 2006, 110, 21497-21508.

(63) Cygan, R. T.; Liang, J.-J.; Kalinichev, A. G. Molecular Models of Hydroxide, Oxyhydroxide, and Clay Phases and the Development of a General Force Field. J. Phys. Chem. B 2004, 108, 1255-1266.

(64) Cox, S. J.; Raza, Z.; Kathmann, S. M.; Slater, B.; Michaelides, A. The microscopic features of heterogeneous ice nucleation may affect the macroscopic morphology of atmospheric ice crystals. Faraday Discuss. 2013, 167, 389-403.

(65) Shahsavari, R.; Pellenq, R. J.-M.; Ulm, F.-J. Empirical force fields for complex hydrated calcio-silicate layered materials. Phys. Chem. Chem. Phys. 2011, 13, 1002-1011.

(66) Kerisit, S. Water structure at hematite-water interfaces. Geochim. Cosmochim. Acta 2011, 75, 2043-2061.

(67) Price, D. J.; Brooks, C. L. A modified TIP3P water potential for simulation with Ewald summation. J. Chem. Phys. 2004, 121, 1009610103.

(68) Wang, J.; Wolf, R. M.; Caldwell, J. W.; Kollman, P. A.; Case, D. A. Development and testing of a general amber force field. J. Comput. Chem. 2004, 25, 1157-1174.

(69) Cole, D. J.; Payne, M. C.; Csányi, G.; Mark Spearing, S.; Colombi Ciacchi, L. Development of a classical force field for the oxidized Si surface: Application to hydrophilic wafer bonding. J. Chem. Phys. 2007, 127, 204704.

(70) Schneider, J.; Ciacchi, L. C. A Classical Potential to Model the Adsorption of Biological Molecules on Oxidized Titanium Surfaces. J. Chem. Theory Comput. 2011, 7, 473-484.

(71) Rustad, J. R.; Wasserman, E.; Felmy, A. R. A molecular dynamics investigation of surface reconstruction on magnetite (001). Surf. Sci. 1999, 432, L583-L588.

(72) Walz, F. The Verwey transition - a topical review. J. Phys.: Condens. Matter 2002, 14, R285-R340.

(73) Wright, J. P.; Attfield, J. P.; Radaelli, P. G. Charge ordered structure of magnetite $\mathrm{Fe}_{3} \mathrm{O}_{4}$ below the Verwey transition. Phys. Rev. B: Condens. Matter Mater. Phys. 2002, 66, 214422.

(74) Hamilton, W. C. Neutron Diffraction Investigation of the $119^{\circ} \mathrm{K}$ Transition in Magnetite. Phys. Rev. 1958, 110, 1050-1057.

(75) Liu, H.; Seifert, G.; Di Valentin, C. An efficient way to model complex magnetite: Assessment of SCC-DFTB against DFT. J. Chem. Phys. 2019, 150, 094703.

(76) Liu, H.; Di Valentin, C. Band Gap in Magnetite above Verwey Temperature Induced by Symmetry Breaking. J. Phys. Chem. C 2017, 121, 25736-25742.

(77) Tabacchi, G.; Fabbiani, M.; Mino, L.; Martra, G.; Fois, E. The Case of Formic Acid on Anatase TiO2(101): Where is the Acid Proton? Angew. Chem., Int. Ed. 2019, 58, 12431-12434.

(78) Henkelman, G.; Uberuaga, B. P.; Jónsson, H. A climbing image nudged elastic band method for finding saddle points and minimum energy paths. J. Chem. Phys. 2000, 113, 9901-9904.

(79) Milonjić, S. K.; Ruvarac, A. L.; Šušíc, M. V. The heat of immersion of natural magnetite in aqueous solutions. Thermochim. Acta 1975, 11, 261-266.

(80) Xantheas, S. S. Cooperativity and hydrogen bonding network in water clusters. Chem. Phys. 2000, 258, 225-231.

(81) Rustad, J. R. Molecular Models of Surface Relaxation, Hydroxylation, and Surface Charging at Oxide-Water Interfaces. Rev. Mineral. Geochem. 2001, 42, 169-198.

(82) Mirabello, G.; Ianiro, A.; Bomans, P. H. H.; Yoda, T.; Arakaki, A.; Friedrich, H.; de With, G.; Sommerdijk, N. A. J. M. Crystallization by particle attachment is a colloidal assembly process. Nat. Mater. 2020, 19, 391-396.

(83) Kawska, A.; Brickmann, J.; Kniep, R.; Hochrein, O.; Zahn, D. An atomistic simulation scheme for modeling crystal formation from solution. J. Chem. Phys. 2006, 124, 024513.

(84) Hueckel, T.; Hocky, G. M.; Palacci, J.; Sacanna, S. Ionic solids from common colloids. Nature 2020, 580, 487-490. 